\title{
Flag Curvature of Invariant Randers Metrics on Homogeneous Manifolds
}

\author{
E. Esrafilian \\ and \\ H. R. Salimi Moghaddam \\ Department of Mathematics, \\ University of Isfahan, \\ Isfahan,81746-73441-Iran. \\ e-mail:
}

salimi.moghaddam@gmail.com and hr.salimi@sci.ui.ac.ir

\begin{abstract}
In this article we review the recent results about the flag curvature of invariant Randers metrics on homogeneous manifolds and by using a counter example we show that the formula which obtained for the flag curvature of these metrics is incorrect. Then we give an explicit formula for the flag curvature of invariant Randers metrics on the naturally reductive homogeneous manifolds $(G / H, g)$, where the Randers metric induced by the invariant Riemannian metric $g$ and an invariant vector field $\tilde{X}$ which is parallel with $g$.
\end{abstract}

AMS 2000 Mathematics Subject Classification: 22E60, 53C60, 53C30.

Key words: Invariant Randers metric, Flag curvature, Reductive homogeneous manifold, Naturally reductive homogeneous manifold.

\section{Introduction.}

The geometry of Finsler manifolds is one of the interesting subjects in differential geometry which found many physical applications (For example see [1] and [2]). One of important quantities which associate to a Finsler metric is the flag curvature which is a generalization of the concept of sectional curvature in Riemannian geometry.

But, in general, the computation of the flag curvature of a Finsler metric is very difficult. Therefore, It is very important to find an explicit and 
applicable formula for the flag curvature. In this article we want to find such explicit formula for the flag curvature of a special type of invariant Randers metrics on homogeneous manifolds.

S. Deng and Z. Hou studied invariant Finsler metrics on reductive homogeneous manifolds and gave an algebraic description of these metrics and obtained a necessary and sufficient condition for a homogeneous manifold to have invariant Finsler metrics (See [4]). Also they studied invariant Randers metrics on homogeneous Riemannian manifolds and used of this structure to construct Berwald space which is neither Riemannian nor locally Minkowskian (For more details see [5]). They gave a formula for the flag curvature of invariant Randers metrics on homogeneous manifolds in $[5]$.

In this paper by using a counter example we show that the formula which obtained in [5] is incorrect. Also we explain why this formula and also an example which gave in [4] are incorrect. Then we give an explicit formula for the flag curvature of invariant Randers metrics on naturally reductive homogeneous manifolds $(G / H, g)$, where the Randers metric induced by the invariant Riemannian metric $g$ and an invariant vector field $\tilde{X}$ which is parallel with $g$.

\section{Preliminaries.}

2.1. Definition. (See[6] or [8]) A homogeneous space $G / H$ of a connected Lie group $G$ is called reductive if the following conditions are satisfied:

(1) In the Lie algebra $\mathfrak{g}$ of $G$ there exists a subspace $\mathfrak{m}$ such that $\mathfrak{g}=\mathfrak{m}+\mathfrak{h}$ (direct sum of vector subspaces).

(2) $\operatorname{ad}(h) \mathfrak{m} \subset \mathfrak{m}$ for all $h \in H$,

where $\mathfrak{h}$ is the subalgebra of $\mathfrak{g}$ corresponding to the identity component $H_{0}$ of $H$ and $a d(h)$ denotes the adjoint representation of $H$ in $\mathfrak{g}$. condition (2) implies

$(2)^{\prime}[\mathfrak{h}, \mathfrak{m}] \subset \mathfrak{m}$

and, conversely, if $H$ is connected, then (2) implies (2).

$G / H$ is reductive in either of the following cases (See[8]):

- $H$ is compact,

- $H$ is connected and semi-simple.

- $H$ is a discrete subgroup of $G ; \mathfrak{h}=0$ and $\mathfrak{m}=\mathfrak{g}$

Let $G / H$ be a reductive homogeneous manifold with invariant Riemannian metric $g$ which the subspace $\mathfrak{m}$ is the orthogonal complement of $\mathfrak{h}$ with 
respect to the inner product on $\mathfrak{g}$. Also let

$$
V=\{X \in \mathfrak{m} \mid \operatorname{ad}(h) X=X,<X, X><1, \forall h \in H\}
$$

Where $<,>$ is the inner product induced by $g$.

Then for any $X \in V$ there exist an invariant Randers metric on $G / H$ by the following formula (See [5]):

$$
F_{X}(x H, y)=\sqrt{g(x H)(y, y)}+g(x H)(\tilde{X}, y), \quad y \in T_{x H}(G / H)
$$

Where $\tilde{X}$ is the corresponding invariant vector field on $G / H$ to $X$.

2.2. Theorem. (See[5]) Let $Y$ be a nonzero vector in $\mathfrak{m}$ and $P$ be a plane in $\mathfrak{m}$ containing $Y$. Then the flag curvature of the flag $(P, Y)$ in $T_{0}(G / H)$ is given by

$$
K(P, Y)=\frac{2 \sqrt{g(Y, Y)}}{2 \sqrt{g(Y, Y)}+g(X, Y)} K(P) .
$$

Where $K(P)$ is the Riemannian curvature of $P$ of the Riemannian metric $g$, and

$$
K(P)=\frac{g\left(\left[[Y, U]_{\mathfrak{h}}, Y\right], U\right)}{g(U, U) g(Y, Y)-g^{2}(U, Y)} .
$$

Where $U$ is any vector in $P$ such that $\operatorname{span}\{Y, U\}=P$ and $[Y, U]_{\mathfrak{h}}$ is the orthogonal projection of $[Y, U]$ to $\mathfrak{h}$.

We will show that the above Theorem is not correct.

\section{Counter example.}

Let $G$ be a connected Lie group and $H=\{e\}$. So $G / H=G$ is a reductive homogeneous manifold with $\mathfrak{m}=\mathfrak{g}$ and $\mathfrak{h}=0$. Also let $g$ be an invariant Riemannian metric on $G / H=G$ and $0=X \in \mathfrak{m}=\mathfrak{g}$.

In this case the invariant Randers metric defined by $g$ and $X$ is Riemannin because:

$$
F_{X}(x H, y)=\sqrt{g(x H)(y, y)}+g(x H)(0, y)=\sqrt{g(x)(y, y)} .
$$

Also we know that if our Finsler metric be Riemannian then the flag curvature reduces to the familiar sectional curvature([3]).

Under the above conditions we have

$$
[Y, U]_{\mathfrak{h}}=0,
$$

because $\mathfrak{h}=0$.

So we have

$$
g\left(\left[[Y, U]_{\mathfrak{h}}, Y\right], U\right)=g([0, Y], U)=g(0, U)=0 .
$$


Therefore $K(P)=0$ and so $K(P, Y)=0$.

By attention to this fact that in this case the flag curvature is the same sectional curvature of $g$, we obtain the following incorrect proposition:

\$ The sectional curvature of all Lie groups with invariant Riemannian metrics is zero.

The above proposition is false. We give the following counter example that show there exist many Lie groups with invariant Riemannian metrics and nonzero sectional curvatures.

3.1. Theorem. (See [10]) Let $G$ be a connected nilpotent Lie group. Let $B$ be a positive definite symmetric bilinear form on the Lie algebra $\mathfrak{g}$ of $G$, and let $M$ be the Riemannian manifold obtained by left translation of $B$ to every tangent space of $G$. Then these are equivalent:

- $M$ has a positive sectional curvature.

- $M$ has a negative sectional curvature.

- $G$ is not commutative.

So if we consider a connected nilpotent noncommutative Lie group $G$ with an invariant Riemannian metric, then it has nonzero sectional curvature. (One can find another counter examples in [7].)

By attention to the above counter example we showed that the proposition

do is incorrect so the Theorem 2.2 is incorrect.

\section{Flag curvature of invariant Randers met- rics on homogeneous manifolds.}

But why the Theorem 2.2 is incorrect?

If we see the proof of this Theorem we see that the authors of the paper [5] use of the formula

$$
R(U, V) W=-\left[[U, V]_{\mathfrak{h}}, W\right]
$$

for the curvature tensor of $F$ (and $g$ ). But the equation 4.2 is a formula for tensor curvature of a special type of invariant affine connections called the canonical affine connection of the second kind (See Theorem 10.3 of [8] or Theorem 2.6 of [6] page 193) which It is not the Riemannian connection. So we can not use of equation 4.2 for the curvature tensor of the Riemannian connection.

Another problem of the proof of Theorem 2.2 is the formula of $g_{Y}(U, V)$. The formula $g_{Y}(U, V)=g(U, V)\left(1+\frac{g(X, Y)}{2 \sqrt{g(Y, Y)}}\right)$ which is used in the proof of Theorem 2.2 is not correct.

The authors of the paper [5] used of the equation 4.2 to compute the flag curvature of an example in the paper [4] (The example after Theorem 
2.1 of the paper [4]). By attention to this fact that the equation 4.2 is not the curvature tensor of the Riemannian connection, so the flag curvature of this example is not correct.

Now by attention to the above discussion, we compute the flag curvature of invariant Randers metrics on homogeneous manifolds.

4.1. Definition. (See [6]) A homogeneous manifold $M=G / H$ with a $G$-invariant indefinite Riemannian metric $g$ is said to be naturally reductive if it admits an $\operatorname{ad}(H)$-invariant decomposition $\mathfrak{g}=\mathfrak{h}+\mathfrak{m}$ satisfying the condition

$$
B\left(X,[Z, Y]_{\mathfrak{m}}\right)+B\left([Z, X]_{\mathfrak{m}}, Y\right)=0 \quad \text { for } X, Y, Z \in \mathfrak{m} .
$$

Where $B$ is the bilinear form on $\mathfrak{m}$ induced by $\mathfrak{g}$ and $[,]_{\mathfrak{m}}$ is the projection to $\mathfrak{m}$ with respect to the decomposition $\mathfrak{g}=\mathfrak{h}+\mathfrak{m}$.

4.2. Theorem. Let $G / H$ be a homogeneous manifold with invariant Riemannian metric $g$ and $F$ be an invariant Randers metric defined by the $\operatorname{ad}(H)$-invariant vector $X$,

$$
F(x H, Y)=\sqrt{g(x H)(Y, Y)}+g(x H)(\tilde{X}, Y) .
$$

Where $g(X, X)<1, Y \in T_{x H} G / H$ and $\tilde{X}$ is the corresponding invariant vector field on $G / H$ to $X$. Also suppose that the vector field $\tilde{X}$ is parallel with respect to $g$ and $(G / H, g)$ is naturally reductive.

Then the flag curvature of the flag $(P, Y)$ in $T_{H}(G / H)$ is given by

$$
K(P, Y)=\frac{A}{B-C} .
$$

Where

$$
\begin{gathered}
A=\quad g(\alpha, U)+g(X, \alpha) \cdot g(X, U)-\frac{g(X, Y) \cdot g(Y, U) \cdot g(Y, \alpha)}{g(Y, Y)^{\frac{3}{2}}} \\
+\quad \frac{1}{\sqrt{g(Y, Y)}}\{g(X, \alpha) \cdot g(Y, U)+g(X, Y) \cdot g(\alpha, U)+g(X, U) \cdot g(Y, \alpha)\} \\
B=\left\{g(Y, Y)+g^{2}(X, Y)+2 g(X, Y) \sqrt{g(Y, Y)}\right\} \times \\
\left\{g(U, U)+g^{2}(X, U)-\frac{1}{\sqrt{g(Y, Y)}}\left\{\frac{g(X, Y) \cdot g^{2}(Y, U)}{g(Y, Y)}\right.\right. \\
+g(X, Y) \cdot g(U, U)+2 g(X, U) \cdot g(Y, U)\}\}
\end{gathered}
$$

and

$$
C=\left\{g(Y, U)\left(1+\frac{g(X, Y)}{\sqrt{g(Y, Y)}}\right)+g(X, U)(g(X, Y)+\sqrt{g(Y, Y)})\right\}^{2} .
$$


Where $U$ is any vector in $P$ such that $\operatorname{span}\{Y, U\}=P$ and in $A$ we have

$$
\alpha=\frac{1}{2}\left[[U, Y]_{\mathfrak{m}}, Y\right]+\left[Y,[Y, U]_{\mathfrak{h}}\right]
$$

$\left([,]_{\mathfrak{m}}\right.$ and $[,]_{\mathfrak{h}}$ are the projections of $[$,$] to \mathfrak{m}$ and $\mathfrak{h}$ respectively.)

Proof. $\tilde{X}$ is parallel with respect to $g$ so by using Lemma 2.1 of [5] $F$ is of Berwald type. Also the Chern connection of $F$ and the Riemannian connection of $g$ coincide (See [3] page 305), therefore we have

$$
R^{F}(U, V) W=R^{g}(U, V) W .
$$

Where $R^{F}$ and $R^{g}$ are the curvature tensors of $F$ and $g$ respectively. (Now let $R:=R^{F}=R^{g}$.)

But $g$ is naturally reductive, so by using Proposition 3.4 of [6] (page 202) we have:

$$
\begin{aligned}
(R(U, V) W)_{0} & =\frac{1}{4}\left[U,[V, W]_{\mathfrak{m}}\right]_{\mathfrak{m}}-\frac{1}{4}\left[V,[U, W]_{\mathfrak{m}}\right]_{\mathfrak{m}} \\
& -\frac{1}{2}\left[[U, V]_{\mathfrak{m}}, W\right]_{\mathfrak{m}}-\left[[U, V]_{\mathfrak{h}}, W\right] \quad \text { for } U, V, W \in \mathfrak{m} .
\end{aligned}
$$

So we have

$$
\begin{aligned}
(R(Y, U) Y)_{0} & =\frac{1}{4}\left[Y,[U, Y]_{\mathfrak{m}}\right]_{\mathfrak{m}}-\frac{1}{4}\left[U,[Y, Y]_{\mathfrak{m}}\right]_{\mathfrak{m}} \\
& -\frac{1}{2}\left[[Y, U]_{\mathfrak{m}}, Y\right]_{\mathfrak{m}}-\left[[Y, U]_{\mathfrak{h}}, Y\right] \\
& =\frac{1}{2}\left[[U, Y]_{\mathfrak{m}}, Y\right]_{\mathfrak{m}}+\left[Y,[Y, U]_{\mathfrak{h}}\right]
\end{aligned}
$$

On the other hand we have:

$$
K(P, Y)=\frac{g_{Y}\left(R_{Y}(U), U\right)}{g_{Y}(Y, Y) \cdot g_{Y}(U, U)-g_{Y}^{2}(Y, U)} .
$$

Where

$$
\begin{aligned}
g_{Y}(U, V) & =\left.\frac{1}{2} \frac{\partial^{2}}{\partial s \partial t}\left\{F^{2}(Y+s U+t V)\right\}\right|_{s=t=0} \\
& =\frac{1}{2} \frac{\partial^{2}}{\partial s \partial t}\{g(Y+s U+t V, Y+s U+t V) \\
& +g^{2}(X, Y+s U+t V) \\
& +2 \sqrt{g(Y+s U+t V, Y+s U+t V)} g(X, Y+s U+t V)\}\left.\right|_{s=t=0}
\end{aligned}
$$

By a direct computation we get

$$
\begin{aligned}
g_{Y}(U, V)= & g(U, V)+g(X, U) \cdot g(X, V)-\frac{g(X, Y) \cdot g(Y, V) \cdot g(Y, U)}{g(Y, Y)^{\frac{3}{2}}} \\
+ & \frac{1}{\sqrt{g(Y, Y)}}\{g(X, U) \cdot g(Y, V) \\
& +g(X, Y) \cdot g(U, V)+g(X, V) \cdot g(Y, U)\} .
\end{aligned}
$$


Therefore

$$
\begin{aligned}
g_{Y}(Y, Y) & =g(Y, Y)+g(X, Y)(g(X, Y)+2 \sqrt{g(Y, Y)}), \\
g_{Y}(U, U) & =g(U, U)+g^{2}(X, U)-\frac{g(X, Y) \cdot g^{2}(Y, U)}{g(Y, Y)^{\frac{3}{2}}} \\
& +\frac{1}{\sqrt{g(Y, Y)}}\{g(X, Y) \cdot g(U, U)+2 g(X, U) \cdot g(Y, U)\}, \\
g_{Y}(Y, U) & =g(Y, U)\left(1+\frac{g(X, Y)}{\sqrt{g(Y, Y)}}\right)+g(X, U)(g(X, Y)+\sqrt{g(Y, Y)}),
\end{aligned}
$$

and

$$
g_{Y}(R(Y, U) Y, U)=g_{Y}(\alpha, U) .
$$

Where $\alpha=\frac{1}{2}\left[[U, Y]_{\mathfrak{m}}, Y\right]_{\mathfrak{m}}+\left[Y,[Y, U]_{\mathfrak{h}}\right]$.

Combining the above formulas with the equation 4.3 completes the proof.

4.3. Note. In the formula obtained in Theorem 4.2 if we assume that $U$ is orthogonal to $Y$ with respect to $g$ then we can obtain a simpler formula.

4.4. Corollary. In a special case we can assume a Lie group $G$ as a reductive homogeneous space with $H=\{e\}, \mathfrak{h}=\{0\}$ and $\mathfrak{m}=\mathfrak{g}$, then our formula for the flag curvature is simpler because in this case we have

$$
\alpha=\frac{1}{2}[[U, Y], Y]
$$

\section{References}

[1] P. L. Antonelli, R. S. Ingarden, M. Matsumoto, The Theory of Sprays and Finsler Spaces with Applications in Physics and Biology, Kluwer Academic Publishers, (1993).

[2] G. S. Asanov, Finsler Geometry, Relativity and Gauge Theories, D. Reidel Publishing Company, (1985).

[3] D. BAo, S. S. Chern, Z. Shen, An Introduction to Riemann-Finsler Geometry, Springer-Verlag, (2000).

[4] S. Deng, Z. Hou, Invariant Finsler Metrics on Homogeneous Manifolds, J. Phys. A: Math. Gen. 37 (2004), 8245-8253.

[5] S. Deng, Z. Hou, Invariant Randers Metrics on Homogeneous Riemannian Manifolds, J. Phys. A: Math. Gen. 37 (2004), 4353-4360.

[6] S. Kobayashi, K. Nomizu, Foundations of Differential Geometry, Volume 2. Interscience Publishers, John Wiley\& Sons (1969).

[7] J. Milnor, Curvatures of Left Invariant Metrics on Lie Groups, Advances in Mathematics. 21 (1976), 293-329. 
[8] K. Nomizu, Invariant Affine Connections on Homogeneous Spaces, Am. J. Math. 76 (1954), 33-65.

[9] Z. Shen, Lectures on Finsler Geometry, World Scientific, (2001).

[10] J. A. Wolf, Curvature in Nilpotent Lie Groups, Proceeding of the Am. Math. Society, Vol. 15, No. 2, (Apr, 1964), 271-274. 